\title{
I so wish it were true... "If it seems too good to be true, it probably is"
}

\author{
Sorin J. Brener \\ Cardiac Catheterization Laboratory, New York - Presbyterian Brooklyn Methodist Hospital, Weill Cornell Medical College, New York, USA
}

Adv Interv Cardiol 2018; 14, 1 (51): 1-4

DOI: https://doi.org/10.5114/aic.2018.74348

Patients presenting with an acute coronary syndrome (ACS) without ST-segment elevation have a high rate of recurrent events in the first 12 months after hospitalization [1]. Thus, any strategy to minimize this rate is highly desirable and could reduce the health and economic burden of $\sim 1.5$ million people in the US alone. Many factors contribute to the adverse prognosis. A high incidence of advanced age, multi-vessel coronary artery disease (MV CAD) and chronic kidney disease characterizes these patients and differentiates them from patients with stable CAD and those with acute ST-segment elevation myocardial infarction (STEMI). As many patients have MV CAD, the optimal method of revascularization has not been fully clarified. Intuitively, more complete revascularization (CR) would seem to be preferred. Yet, there has not been a randomized clinical trial addressing this clinical scenario and many analyses of selected populations have provided somewhat conflicting results.

Why would then a common-sense approach not show the expected results? Many reasons have been cited, suggesting maybe that we do not quite know the answer. It is likely that some of the lesions treated did not require intervention (functionally not significant), while some of those treated had irreversibly dysfunctional myocardium. It is also possible that many of the non-culprit lesions do not cause death, recurrent infarction or ACS leading to reintervention - the very same endpoints captured in this analysis - but rather manifest as stable angina and lower quality of life.

In this issue of Advances in Interventional Cardiology, Hawranek et al. examined this very topic in a large cohort of such patients [2]. Out of more than 1,500 ACS patients treated over 9 years, 695 ( $44 \%, 70 \%$ of whom had a final diagnosis of myocardial infarction) qualified for the study, but we cannot tell what were the main reasons for exclusion, among the list of potential disqualifying character- istics. Nearly $20 \%$ of them had complete revascularization (mainly in one stage), while the rest did not. Such an imbalance in the treatment of interest would require complex statistical modeling (such as inverse probability of treatment weighting propensity score) in an attempt to adjust for the obvious differences between the two groups. The authors chose not to do so and applied only a rather conventional multivariable model. The endpoints of interest were the composite of death, non-fatal myocardial infarction and revascularization in the setting of recurrent ACS at 30 days and at 12 months, without independent adjudication of the events. It seems that CR was assessed based on anatomic considerations only. The patients in the two groups were different from each other - such that the lower risk patients (predominantly 2-vessel CAD, higher ejection fraction, better kidney function, no advanced heart failure, less chronic total occlusions, etc.) were assigned to $C R$, while the worse off ones received incomplete revascularization (IR) and were probably not bypass surgery candidates. The results were both surprising and comforting. On one hand, CR - achieved, incredibly, in $100 \%$ of patients selected for it - was not associated with a higher rate of early adverse events - such as in-hospital death, myocardial infarction (MI), bleeding or acute kidney injury. At 30 days, CR was associated with a massive $69 \%$ reduction in the primary endpoint and an even more difficult to believe $89 \%$ reduction in death. Complete revascularization was an independent predictor of freedom from the primary endpoint and death at this interval, even after adjusting for many potentially influencing covariates, in a probably over fitted model for the $\sim 60$ primary events that occurred. At 1 year, there was a robust reduction of $44 \%$ in the composite endpoint in favor of CR (unadjusted), which was again an independent predictor of freedom from the primary endpoint (50\% unadjusted reduction and $54 \%$ adjusted reduction), but not of all-cause mortal-

\section{Corresponding author:}

Prof. Sorin J. Brener MD, PhD, Cardiac Catheterization Laboratory, New York - Presbyterian Brooklyn Methodist Hospital, Weill Cornell Medical College, NY 11215, USA, phone: +17 18780 5143, fax: +1718 780 3930, e-mail: sjb9005@nyp.org Received: 21.12.2017, accepted: 21.12.2017. 


\begin{tabular}{lcccc} 
& Events & Total & Events & Total \\
\hline Retrospective & & & & \\
Abe (2013) & 24 & 220 & 17 & 54 \\
Corpus (2004) & 42 & 354 & 5 & 26 \\
Hannan (2010) & 14 & 259 & 36 & 503 \\
lqbal (2014) & 255 & 3429 & 56 & 555 \\
Mohamad (2011) & 3 & 30 & 2 & 7 \\
Qarawani (2008) & 2 & 25 & 9 & 95 \\
Roe (2001) & 10 & 61 & 17 & 68 \\
Fixed effect model & & $\mathbf{4 3 7 8}$ & & $\mathbf{1 3 0 8}$
\end{tabular}

\section{Random effects model}

Heterogeneity: $I^{2}=18 \%, \tau^{2}=0.03, p=0.29$

\section{Prospective}

Di Mario (2004)

Dziewierz (2010)

Gershlick 92015)

Jeger (2014)

Politi (2010)

Wald (2013)

Fixed effect model

$\begin{array}{cc}0 & 17 \\ 57 & 707 \\ 6 & 146 \\ 40 & 1467 \\ 13 & 84 \\ 16 & 231 \\ & 2652\end{array}$

Random effects model

Heterogeneity: $R^{2}=40.8 \%, \tau^{2}=0.16, p=0.13$

Fixed effect model

7030

Random effects model

Heterogeneity: $I^{2}=40.9 \%, \tau^{2}=0.11, p=0.06$

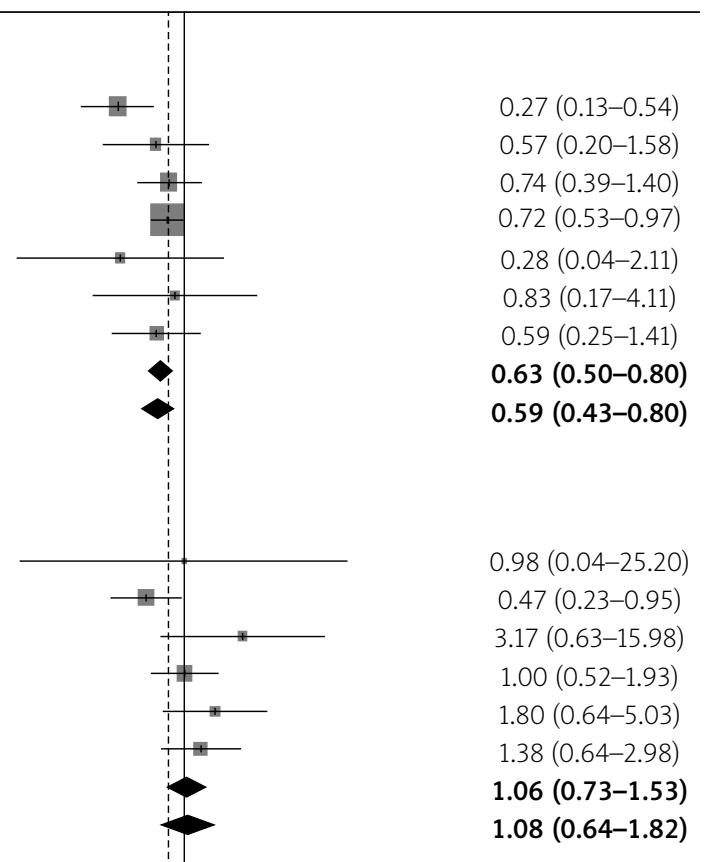

2321

$0.74(0.61-0.90)$

$0.73(0.54-0.99)$

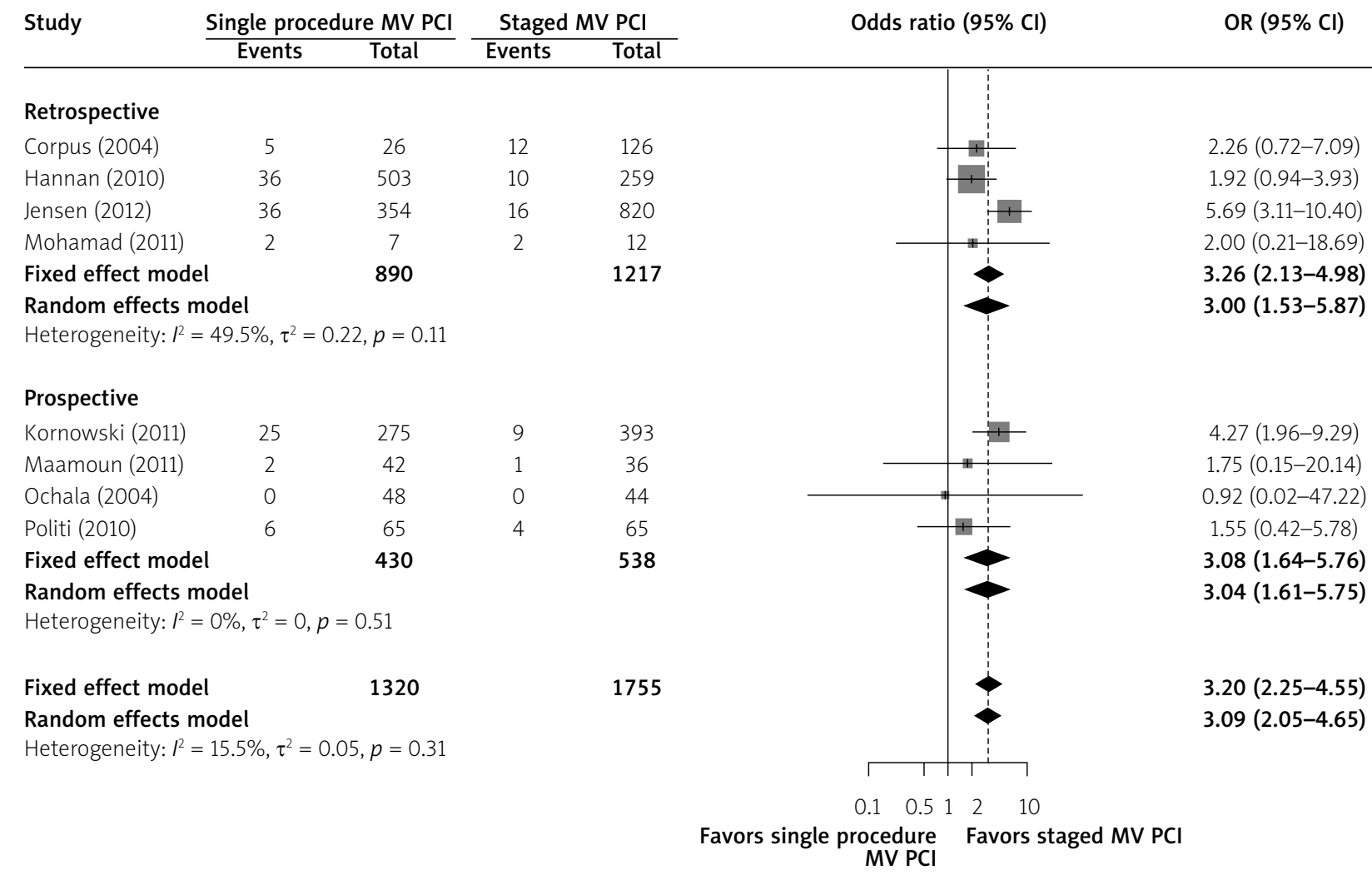

Figure 1. Long-term outcome for various strategies of revascularization in acute ST-segment elevation myocardial infarction (from [3] - Tarantini et al.) 


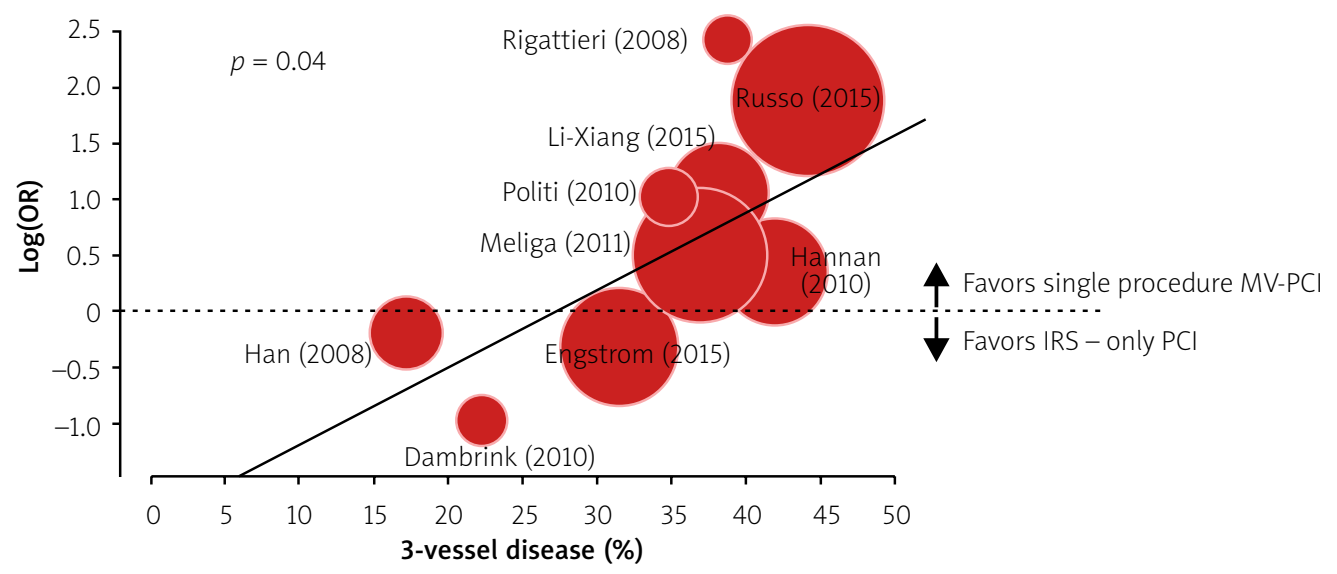

Figure 2. Meta-regression analysis of benefit of staged multi-vessel PCl (from [3] - Tarantini et al.)

ity (adjusted reduction of $22 \%, p=0.56$ ). There were no differences in $\mathrm{MI}$ or revascularization related to ACS. The authors do not offer in my opinion a plausible biological explanation for these large differences in outcome, larger than any benefit seen for primary percutaneous coronary intervention $(\mathrm{PCI})$ in STEMI compared with lytic therapy or even placebo. As expected, CR was predicted by lower acuity CAD (6-fold in favor of 2-vessel CAD), lack of chronic total occlusions (100-fold difference) and better kidney function.

I cannot avoid being skeptical of the reproducibility of these data and the magnitude of the observed benefit, as I consider what we know from other attempts to address this thorny issue. There are no randomized studies on complete vs. incomplete revascularization in ACS, but one can appropriate data from STEMI studies on this subject, considering that those patients may potentially benefit even more than ACS patients. In a pairwise and network meta-analysis of 32 prospective $(n=13)$ and retrospective $(n=19)$ studies with 54,148 patients presenting with MV CAD and STEMI, we found that staged MV PCI had significantly better short- and long-term outcomes, compared with single-procedure $\mathrm{PCl}$ or infarct-related $\mathrm{PCI}$ only (Figure 1). The greatest benefit in meta-regression analysis was in patients with 3-vessel CAD and diabetes (Figure 2) - the opposite of what was observed in this study [3]. Even in the ACUITY study analysis of CR, the mechanism for reduction in major adverse cardiac events was fewer MIs and less repeat revascularization, features not present in this analysis. Using a cohort of patients with ACS treated between 1995 and 2005, Shishehbor et al. found among 1,240 ACS patients treated with $\mathrm{PCl}$ (all bare-metal stents) that 479 (38.6\%) patients had MV PCI, roughly twice the frequency as in the current paper. After adjusting for baseline characteristics and propensity score for more complete revascularization, $\mathrm{MV} \mathrm{PCI}$ was associated with a lower rate of death, $\mathrm{MI}$ or revascularization at 2 years (hazard ratio: $0.67 ; 95 \%$ confidence interval 0.51 to 0.88 ; $p=0.004)$. All the difference between the groups was driven by a lower rate of repeat revascularization, without a reduction in death or $\mathrm{MI}$ [4].
Another way of examining this issue is to evaluate the relationship between the extent of unrevascularized CAD and outcomes. The residual SYNTAX (Synergy Between $\mathrm{PCl}$ with Taxus and Cardiac Surgery) score (rSS) has been instrumental in this area and was studied extensively. Genereux et al. measured the baseline and residual SYNTAX score in 2,668 patients enrolled in the ACUITY study using a central (core) angiographic laboratory [5]. Age, insulin-treated diabetes, hypertension, smoking, elevated biomarkers or ST-segment deviation, and lower ejection fraction were more frequent in patients with IR compared with CR. The 30-day and 1-year rates of ischemic events were significantly higher in the IR group compared with the CR group, especially those with a high rSS. In multivariable analysis, rSS was a strong independent predictor of all ischemic outcomes at 1 year, including all-cause mortality (hazard ratio: $1.05,95 \%$ confidence interval: 1.02 to 1.09 , $p=0.006)$. These data suggest the benefit of more complete revascularization, but to a much lesser degree than in the current analysis. It is notable that when physiologically examined with fractional flow reserve only one third to one half of non-culprit lesions are hemodynamically significant $[6,7]$.

Thus, while it is logical and possible that MV PCI for patients with acute coronary syndromes improves outcome, the large selection bias in most reported series precludes drawing definitive conclusions with potential to affect guidelines of practice. Until a randomized clinical trial is performed, the data presented in this paper remain too good to be true.

\section{Conflict of interest}

The author declares no conflict of interest.

\section{References}

1. van Domburg RT, van Miltenburg-van Zijl AJ, Veerhoek RJ, Simoons ML. Unstable angina: good long-term outcome after a complicated early course. J Am Coll Cardiol 1998; 31: 1534-9. 
2. Hawranek M, Desperak P, Gąsior P, et al. Early and long-term outcomes of complete revascularization with percutaneous coronary intervention in patients with multivessel cronary artery disease presenting with non-ST-segment elevation acute coronary syndromes. Adv Interv Cardiol 2018; 14: 32-41.

3. Tarantini G, D'Amico G, Brener SJ, et al. Survival after varying revascularization strategies in patients with ST-segment elevation myocardial infarction and multivessel coronary artery disease: a pairwise and network meta-analysis. JACC Cardiovasc Interv 2016; 9: 1765-76.

4. Shishehbor MH, Lauer MS, Singh IM, et al. In unstable angina or non-ST-segment acute coronary syndrome, should patients with multivessel coronary artery disease undergo multivessel or culprit-only stenting? J Am Coll Cardiol 2007; 49: 849-54.

5. Genereux P, Palmerini T, Caixeta A, et al. Quantification and impact of untreated coronary artery disease after percutaneous coronary intervention: the residual SYNTAX (Synergy Between PCI with Taxus and Cardiac Surgery) score. J Am Coll Cardiol 2012; 59: 2165-74.

6. Smits PC, Abdel-Wahab M, Neumann FJ, et al. Fractional flow reserve-guided multivessel angioplasty in myocardial infarction. N Engl J Med 2017; 376: 1234-44.

7. Layland J, Oldroyd KG, Curzen N, et al. Fractional flow reserve vs. angiography in guiding management to optimize outcomes in non-ST-segment elevation myocardial infarction: the British Heart Foundation FAMOUS-NSTEMI randomized trial. Eur Heart J 2015; 36: 100-11. 